\title{
Internações por câncer de mama feminino na região metropolitana de Porto Alegre
}

RESUMO | Objetivo: caracterizar as internações por câncer de mama feminino na rede pública da Região Metropolitana de Porto Alegre de 2016 a 2018. Método: estudo epidemiológico de base populacional, observacional, transversal e retrospectivo com análise de dados secundários em saúde acessados no Sistema de Informações Hospitalares do Sistema Único de Saúde das internações com diagnóstico de câncer de mama. As variáveis extraídas foram internações por faixa etária, raça/cor, tempo de permanência, óbitos, taxa de mortalidade e gasto hospitalar. Resultados: Nesse período ocorreram 7.049 internações, 3.807(54\%) entre 50 a 69 anos. A média de permanência na internação foram 4 dias e o custo médio total/ano pelo SUS de 375.374,604 reais. No triênio ocorreram 504 (7,15\%) pela doença. Conclusão: As internações por câncer de mama em mulheres na RMPA repercutem diretamente no aumento de gastos e no mais prolongado uso de leitos hospitalares, tornando a assistência a esse tipo de agravo cada vez mais oneroso.

Palavras-chaves: Câncer da mama;Internações; Saúde Pública; Epidemiologia.

\begin{abstract}
Objective: to characterize hospitalizations for female breast cancer in the public network of the Metropolitan Region of Porto Alegre from 2016 to 2018. Method: population-based epidemiological study, observational, cross-sectional and retrospective study with analysis of secondary health data accessed in the Hospital Information System of the Unified Health System of hospitalizations with a diagnosis of breast cancer. The extracted variables were hospitalizations by age group, race/ color, length of stay, deaths, mortality rate and hospital expenditure. Results: In this period, 7,049 hospitalizations occurred, $3,807(54 \%)$ between 50 and 69 years of age. The average stay in hospital was 4 days and the average total cost/year by SUS was $375,374.604$ reais. In the triennium there were 504 (7.15\%) due to the disease. Conclusion: Hospitalizations for breast cancer in women in the RMPA have a direct impact on thein crease in expenses and on the longer use of hospital beds, making assistance to this type of disease increasingly costly.
\end{abstract}

Keywords: Breast cancer; Hospitalizations; Public health; Epidemiology.

RESUMEN | Objetivo: caracterizar lashospitalizaciones por cáncer de mama femeninoenlared pública de laRegión Metropolitana de Porto Alegre de 2016 a 2018. Método: estudio epidemiológico poblacional, observacional, transversal y retrospectivo conanálisis de datossecundarios de salud a los que se accedeenel Sistema de Información Hospitalaria del Sistema Único de Salud de hospitalizacionescon diagnóstico de cáncer de mama. Lasvariables extraídas fueronhospitalizaciones por grupo de edad, raza/ color, tiempo de estancia, defunciones, tasa de mortalidad y gasto hospitalario. Resultados: En este período ocurrieron 7.049 hospitalizaciones, 3.807 (54\%) entre 50 y 69 años. La estadíapromedioenel hospital fue de 4 días y elcosto total promedio/año por el SUS fue de 375.374,604 reales. Eneltrieniohubo 504 (7,15\%) debido a laenfermedad. Conclusión: Lashospitalizaciones por cáncer de mama enmujeres de la RMPA tienenun impacto directoenel aumento de los gastos y enelmayor uso de lascamas hospitalarias, haciendo que laatención a este tipo de enfermedadessea cada vez más costosa.

Palabras claves: Cáncer de mama; Hospitalizaciones; Salud pública; Epidemiología.

\section{Daiana Almeida da Silva}

Enfermeira assistencial Hospital Mãe de Deus.

ORCID: 0000-0002-5767-9822

\section{Ataliba Antonio da Silva das Neves}

Enfermeiro. Centro Universitário Ritter dos Reis.

ORCID: 0000-0003-0393-1647

\section{Thainá Dias Luft \\ Acadêmica de enfermagem do Centro Universitário Ritter Dos Reis (UNIRITTER). ORCID: 0000-0002-3690-2546}

\section{Gabriela Oliveira Zavaglia}

Enfermeira pesquisadora do Hospital Moinhos de Vento.

ORCID: 0000-0001-5419-665X

\section{Nathalia Torres Mendes}

Enfermeira do Hospital São Lucas da PUC-RS e pós graduanda em CTI adulto e assistência ao paciente crítico na Facudade Dom Alberto.

ORCID: 0000-0002-2321-818X

\section{Luciana Medeiros Paungartner}

Pesquisadora Voluntária do Programa de Pediatria e Saúde da Criança da PUCRS. Enfermeira - Pós Graduanda em UTI Geral e Gestão da Assistência Intensiva ao Paciente Crítico - Instituto Faveni. ORCID:0000-0003-1983-1114

Recebido em: $18 / 12 / 2020$

Aprovado em: 07/01/2021

\section{Amanda Pereira Ferreira Dellanhese}

Enfermeira. Doutora em Saúde da Criança e do Adolescente pela Universidade Federal do Rio Grande do Sul. Docente Colaboradora da Residência Integrada Multiprofissional em Saúde Coletiva da Universidade Federal do Rio Grande do Sul.

ORCID: 0000-0002-1515-9693

\section{Morgana Thaís Carollo Fernandes}

Enfermeira. Pós-Doutoranda em Saúde da Criança pela Pontifícia Universidade Católica do Rio Grande do Sul (PUCRS), bolsista pela Universityof Toronto (UofT). Professora Colaboradora da Residência Multiprofissional em Saúde da Criança e Pesquisadora Associada do Programa de Extensão e Pesquisa em Saúde Urbana, Ambiente e Desigualdades da Universidade Federal do Rio Grande do Sul (UFRGS). ORCID:0000-0002-7989-294X 
INTRODUÇÃO

A s doenças e agravos não transmissíveis (DANT) já são as principais responsáveis pelo adoecimento e óbito da população no mundo, principalmente nos países de baixo e médio desenvolvimento, com destaque para as cardiovasculares e o câncer. As neoplasias malignas, as transições demográficas e epidemiológicas globais sinalizam um impacto cada vez maior na carga da doença nas próximas décadas ${ }^{1}$.

De acordo com a InternationalAgency for ResearchonCancer (IARC), após o câncer de pele não melanoma, o câncer de mama é o mais incidente em mulheres no mundo, representando $24,2 \%$ do total de casos de câncer feminino com incidência em 2018. A quinta causa de morte por câncer em geral e a mais frequente em mulheres com taxa de mortalidade de 18,9 a cada 100 mil habitantes ${ }^{1}$.

A magnitude dessa doença no Brasil representa um ponto relevante de atenção à gestão pública. As projeções do Ministério da Saúde para o triênio 20202022 são de que surjam 66.280 novos casos, correspondendo a um risco estimado de 61 novos casos a cada 100 mil mulheres $^{2}$. Ainda, as taxas de mortalidade no Brasil seguem elevadas e correspondem aproximadamente a 13/100.000 óbitos em 2018 ${ }^{3}$.Em análises inter-regionais, percebem-se diferenças entre as taxas de incidência, sendo o Sul a região com a maior incidência com risco estimado de 7,06 a cada 100 milhabitantes ${ }^{1}$.

Em países em desenvolvimento como o Brasil, o diagnóstico e tratamento tardio do câncer de mama pode refletir uma redução na sobrevida das pessoas diagnosticadas de até cinco anos, representando as falhas mais significativas na abordagem do câncer de mamarelacionadas ao diagnóstico e tratamento, em comparação com países desenvolvidos (50 a $60 \%$ contra $85 \%)^{4}$.

Uma pesquisa anterior se propôs a estudar as hospitalizações no Sistema único de Saúde (SUS) por essa neoplasia constatou que a análise dos valores hospitala- res é um fator importante para identificar recursos finitos e escassos em saúde, definindo quais regiões e procedimentos são mais e menos frequentes, informações essas que são fundamentais para a distribuição dos recursos em saúde ${ }^{5}$.

Intensificar análises epidemiológicas inter-regionais sobre o câncer de mama feminino, a fim de subsidiar medidasde avaliação e prevenção efetivas, bem como, a qualificação do planejamento de políticas de saúde que visem à redução do impacto da doença na população, compreensão do comportamento e variáveis envolvidas para subsidiar o planejamento, implementação e avaliação das políticas e ações do SUS voltadas à essa doença devem ser estimuladas. O objetivo desse estudo foi caracterizar as internações por câncer de mama feminino na Rede Pública da Região Metropolitana de Porto Alegre entre 2016 e 2018.

\section{MÉTODO}

Trata-se de um estudo de caráter epidemiológico de base populacional, observacional, transversal e retrospectivo com análise de dados secundários em saúde acessados no Sistema de Informações Hospitalares do Sistema único de Saúde (SIH-SUS). A obtenção dos dados se deu por meio de consulta aos arquivos públicos do SIH/SUS disponíveis na base de dados do Departamento de Informática do Sistema Único de Saúde no Brasil (DATASUS), em formato RD'

\begin{tabular}{l|c|c|}
$\begin{array}{l}\text { Quadro 1 - Causas de internação avaliadas no estudo. } \\
\text { Grupo }\end{array}$ & \multicolumn{1}{|c|}{ Diagnóstico } & CID 10 \\
\hline \multirow{4}{*}{$\begin{array}{l}\text { Neoplasia maligna } \\
\text { da mama }\end{array}$} & Neoplasia maligna da porção central da mama & C50.1 \\
\cline { 2 - 3 } & Neoplasia maligna do quadrante inferior interno da mama & C50.3 \\
\cline { 2 - 3 } CID10: C50 & Neoplasia maligna do quadrante superior externo da mama & C50.4 \\
\cline { 2 - 3 } & Neoplasia maligna do quadrante inferior externo da mama & C50.5 \\
\cline { 2 - 3 } & Neoplasia maligna da porção axilar da mama & C50.6 \\
\cline { 2 - 3 } & Neoplasia maligna da mama com lesão invasiva & C50.8 \\
\cline { 2 - 3 } & Neoplasia maligna da mama, não especificada & C50.9 \\
\hline
\end{tabular}

O diagnóstico de internação avaliado no estudo, considerado na Autorização de Internação Hospitalar (AIH),foi o grupo C50 do Código Internacional de Doenças (CID-10), neoplasia maligna da mama conforme detalhado abaixo no Quadro ${ }^{7}$.

Para a análise descritiva foram suprimidas as internações do sexo masculino devido à baixa representatividade e por não se tratar do foco deste estudo. As variáveis extraídas foram: internações por faixa etária, raça/cor, tempo de permanência, óbitos, taxa de mortalidade e gasto hospitalar.

O SIH/SUS utiliza como principal instrumento de coleta de dados a Autorização de Internação Hospitalar (AIH) que apresenta dois modelos: (i) $\mathrm{AlH}-1$, ou Tipo Normal e a (ii) AlH-5, ou Tipo Longa Permanência, para dados de pacientes crônicos ou psiquiátricos que necessitam de continuidade de tratamento8. O período de competência de processamento foi igual ao mês anterior da apresentação da $\mathrm{AlH}$ para faturamento que corresponde, geralmente, ao mês da alta.

Os dados foram extraídos no período de julho e agosto de 2020, utilizando dois tabuladores disponibilizados pelo Ministério da Saúde: o sistema TabNET, que realiza cruzamentos de variáveis básicas diretamente na internet e o TabWINpara tabulações mais avançadas sobre os arquivos capturados. A análise dos dados foi realizada no Microsoft Excel $(\mathbb{R}$ e apresentada de forma descritiva e comparativa em frequência absoluta e relativa categorizada por faixa etária e raça/cor.
Fonte: Brasil/MS. Portaria $\mathrm{n}^{\circ}$ 221, de 17 de abril de 2008 
Quanto aos aspectos éticos, os arquivos do SIH/SUS são de domínio público, disponíveis na internet e divulgados pelo Ministério da Saúde em formato que preserva a identificação dos sujeitos, garantindo a confidencialidade. De acordo com a Resolução 510/2016-Conselho Nacional de Saúde, o estudo não está sujeito ao sistema CEP/CONEP ${ }^{4}$.

\section{RESULTADOS E DISCUSSÃO}

Este estudo possibilitou caracterizar as internações por câncer de mama feminino, na Rede Pública da Região Metropolitana de Porto Alegre (RMPA), entre os anos de 2016 a 2018 permitindo identificar 7.049 registros de internações no triênio avaliado.

Observou-se que em 2016 o maior número deinternações, ocorreu na faixa etária de 50 a 59 anos, perfazendo $28,48 \%$ do total de casos de pacientes que internaram pela doença, seguido da faixa etária de 60 a 69 anos representando 25,55\%. Ainda em relação ao perfil da mulher hospitalizada por câncer de mama na RMPA, quando observada à raça/cor, a branca foi predominante na amostra, com $82,54 \%$, seguida pela preta com $7,16 \%$. Na tabela 1 encontra-se o detalhamento por faixa etária e raça/cor da frequência de internações por câncer de mama feminino registrado.

Tabela 1-Internações por Câncer de Mama Feminino segundo Faixa Etária e Raça/ cor na Região Metropolitana de Porto Alegre entre 2016 e 2018.

\begin{tabular}{|c|c|c|c|c|c|c|c|c|c|c|}
\hline \multirow[t]{2}{*}{ Faixa Etária } & \multicolumn{2}{|c|}{ Branca } & \multicolumn{2}{|c|}{ Preta } & \multicolumn{2}{|c|}{ Parda } & \multicolumn{2}{|c|}{ Amarela } & \multicolumn{2}{|c|}{ Total } \\
\hline & $\mathrm{N}^{0}$ & $\%$ & $\mathrm{~N}^{\circ}$ & $\%$ & $\mathrm{~N}^{\circ}$ & $\%$ & $\mathrm{~N}^{\circ}$ & $\%$ & $\mathrm{~N}^{\circ}$ & $\%$ \\
\hline 15 a 20 anos & 9 & 0,13 & 1 & 0,01 & - & - & - & - & 12 & 0,17 \\
\hline 20 a 29 anos & 69 & 0,98 & 9 & 0,13 & 2 & 0,03 & - & - & 94 & 1,33 \\
\hline 30 a 39 anos & 449 & 6,37 & 50 & 0,71 & 13 & 0,18 & 3 & 0,04 & 559 & 7,93 \\
\hline 40 a 49 anos & 1.197 & 16,68 & 92 & 1,31 & 34 & 0,48 & 4 & 0,06 & 1.423 & 20,19 \\
\hline 50 a 59 anos & 1.622 & 23,01 & 169 & 2,40 & 59 & 0,84 & 6 & 0,09 & 2.006 & 28,46 \\
\hline 60 a 69 anos & 1.497 & 21,24 & 122 & 1,73 & 37 & 0,52 & 1 & 0,01 & 1.801 & 25,55 \\
\hline 70 a 79 anos & 753 & 10,68 & 50 & 0,71 & - & - & - & - & 883 & 12,53 \\
\hline 80 anos ou mais & 222 & 3,15 & 12 & 0,71 & 3 & 0,04 & - & - & 271 & 3,84 \\
\hline Total & 5.818 & 82,54 & 505 & 7,16 & 160 & 2,27 & 14 & 0,20 & 7.049 & 100 \\
\hline
\end{tabular}

Fonte: Ministério da Saúde - Sistema de Informações Hospitalares do SUS (SIH/SUS). Observação: suprimiu-se 552 internações em que não se tinha informações sobre raça/cor e faixa etária.

Outro estudo realizado pelo Instituto Nacional do Câncer (INCA) com base nos Registros Hospitalares de Câncer (RHC), que são disponíveis nos hospitais que fazem parte da rede de atenção especializada em Oncologia no SUS, descreve que no período de 2013 a 2015 foram registradas 67.733 mulheres que chegaram ao hospital sem diagnóstico ou tratamento, com idade mediana de 55 anos para as que realizaram a primeira consulta para o tratamento da neoplasia ${ }^{2}$.

Diretrizes para a detecção precoce do câncer de mama no Brasil recomendam a identificação através de ações de rastreamento e diagnóstico precoce por meio de mamografia bienal, para mulheres com faixa etária entre 50 a 69 anos e exame clínico das mamas para mulheres a partir dos 40 anos de idade 4 . Tais medidas contribuem com um melhor prognóstico, mitigando o impacto da doença e otimizando o planejamento terapêutico ${ }^{2}$.

Contudo, as informações disponíveis sobre o cenário do câncer de mama revelam preocupação no que se refere à prevenção primária no Brasil, devido ao aumento da frequência de fatores de risco e a diminuição dos fatores de proteção, que não alcançam os níveis de promoção de saúde recomendados ${ }^{2}$.As consequências dessas falhas evidenciam o diagnóstico tardio do câncer de mama, 


\section{Figura 1 - Óbitos por Câncer de Mama Feminino segundo raça/cor na Região} Metropolitana de Porto Alegre entre 2016 e 2018.

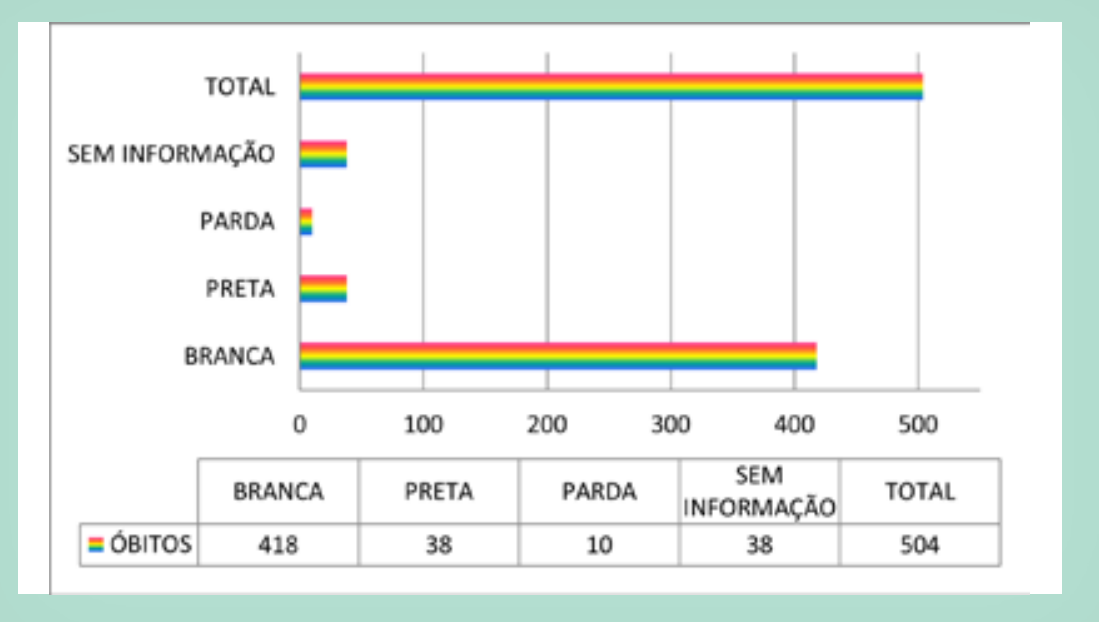

Fonte: Ministério da Saúde - Sistema de Informações Hospitalares do SUS (SIH/SUS).

Figura 2 - Óbitos por Câncer de Mama Feminino segundo Faixa etária na Região Metropolitana de Porto Alegre entre 2016 e 2018.

\section{ÓBITOS}

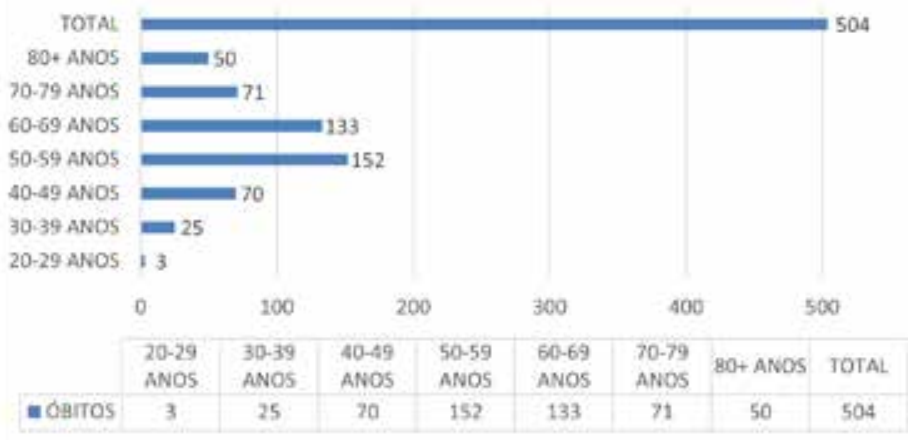

Fonte: Ministério da Saúde - Sistema de Informações Hospitalares do SUS (SIH/SUS).Observação: a faixa etária de 15 a 19 anos foi suprimida porque não apresentaram óbitos.

ressaltou o aumento na mortalidade na faixa etária de 20 a 60 anos, ao decorrer dos 13 anos analisados. O grupo etário que sofreu a maior variação foi de 20 a 39 anos, aumentando em $2,11 \%$, seguida pela de 60 anos ou mais, aumentado em 1,96\% ${ }^{9}$.

Por ser o câncer de mama um dos maiores causadores de morte no Brasil, é importante o reconhecimento por parte dos gestores de saúde, da qualidade do atendimento no território, os recursos disponíveis e as demandas dos usuários cadastrados. A incorporação do mapeamento de fluxo geográfico proporciona a contribuição na identificação de áreas de risco para o câncer e no direcionamento das ações de saúde ${ }^{13}$.

Destaca-se que as taxas de mortalidade por câncer de mama são diretamente relacionadas ao acesso de mulheres ao serviço de saúde e à qualidade na assistência. A neoplasia mamária confere um desafio para os setores de saúde, uma vez que, os programas de rastreio, detecção precoce e tratamento enfrentam dificuldade para garantir a assistência a toda populaçãoe isso pode ser identificado neste estudo, por meio do alto número de in- ternações de usuários em municípios que não aqueles de sua procedência ${ }^{1}$.

Constatou-se nessa pesquisa que uma internação por câncer de mama possui duração média de 4 dias, onde a maior permanência hospitalar está entre a faixa etária de 80 anos ou mais com 5,5 dias em média. $\mathrm{O}$ valor total gasto nas internações por câncer de mama na RMPA foi de $R \$ 13.513 .522,46$ com média anual de R\$ 375.375,624 reais. A faixa etária com maior gasto médio por internação foi entre 20 a 29 anos de idade com $\mathrm{R} \$ 2.552,27$ reais.Ainda, no período do estudo ocorreram 3210 hospitalizações para cirurgias da mama sendo 1027 oncológicas. O valor total envolvido nestes procedimentos foi de 1.887.282,87, sendo 453.941,73 com profissionais 6 .O gráfico 1 apresenta a média de permanência por faixa etária em comparação com o valor médio das internações no triênio 2016-2018.

O Observatório de Oncologia reportou em 2016 que os gastos por pacientes com câncer em internação entre 1990 e 2015 tiveram aumento de $\mathrm{R} \$ 470$ milhões para R\$ 3,3 bilhões. Quanto ao câncer de mama o custo foi de $R \$ 315.760,62$ e identificou-se aumento dos valores em estágios mais avançados da doença e em mulheres pós-menopausa. No Brasil, o Tribunal de Contas da União reportou em 2010 que 60,5\% dos casos de câncer mamário feminino são diagnosticados em estágios III e IIII, com adjeção entre $60 \%$ a 80\% e pior prognóstico.

Esse achado corrobora com o fato de a assistência à saúde em grandes centros urbanos, ainda, ser desafio para os gestores. Sendo assim, a qualificação e a melhor utilização dos recursos especializados, tais como encaminhamentos para os centros de saúde, melhor organização dos fluxos e integração das redes, ajustes na relação entre referência e contra referência nos municípios, auxiliaria na garantia da atenção integral, englobando os procedimentos especializados e redes de apoio para diagnósticos e tratamentos ${ }^{14}$. Este fato pode estar relacionado à dificuldade da construção de redes de atenção à saúde que alcancem 
Figura 3. Comparação da média de permanência por faixa etária e valor médio das internações por Câncer de Mama Feminino na Região Metropolitana de Porto Alegre entre 2016 e 2018.

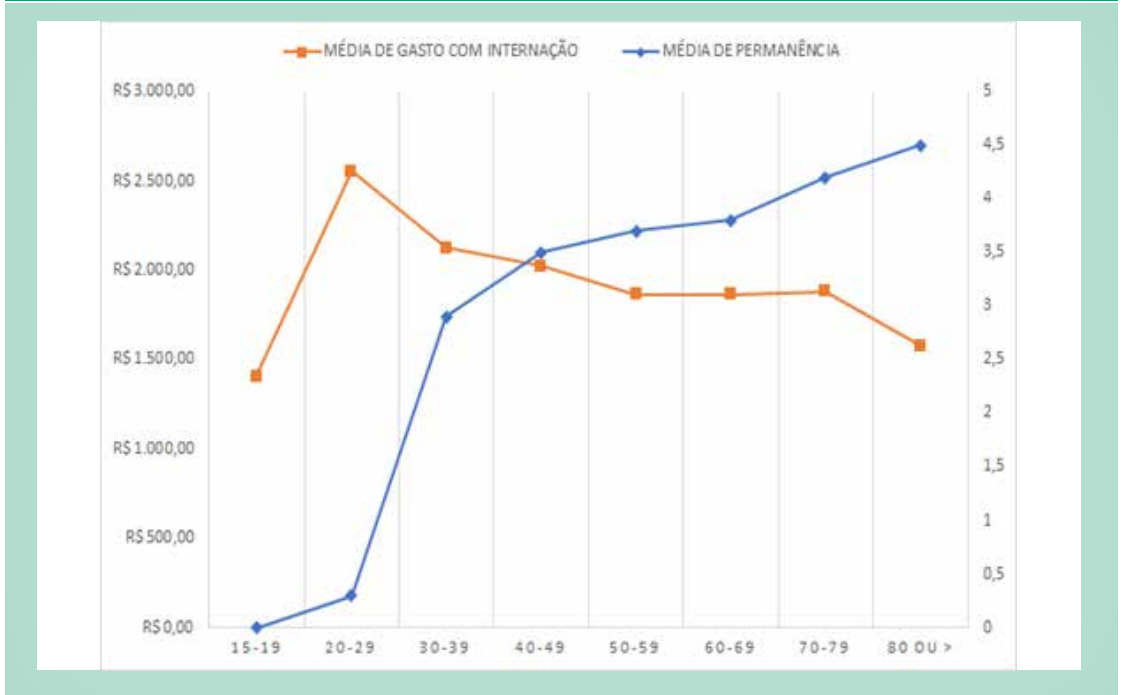

Fonte: Ministério da Saúde - Sistema de Informações Hospitalares do SUS (SIH/SUS).

municípios com baixa densidade tecnológica $^{15}$, como os inseridos em regiões periféricas e grandes centros urbanos.

As estratégias para o controle das neoplasias malignas no país enfrentam problemas que afetam desde as formulações de políticas, até o desenvolvimento de ações e serviços em prol da sociedade, representando um desafio para o Sistema Único de Saúde (SUS) por demandar organização e eficiência na garantia do acesso da população aos serviços de saúde para diagnóstico e tratamento ${ }^{10}$.

Apesar das limitações do uso de estudos ecológicos com dados secundários em saúde, especialmente em relação ao Sistema de Informação hospitalar, dado os problemas de preenchimento das Autorizações de Internação Hospitalar, essa metodologia vem sendo cada vez mais utilizada em pesquisas na área da saúde. Para a presente pesquisa foi utilizado o período com 2016 a 2018 por representar os dados mais atuais disponíveis na plataforma.

\section{CONCLUSÃO}

Conclui-se que das 7.049 mil internações no período avaliado, 54\% encontravam-se na faixa etáriaentre 50 a 69 anos e os óbitos de mulheres internadas por câncer correspondeu a504 (7,15\%). A média de permanência na internação foi de 4 diase a de gastos anual pelo Sistema Único de Saúde foi de 375.374,604 reais apresentandoa faixa etária com maior gasto médio por internação entre 20 a 29 anos de idade com $\mathrm{R} \$ 2.552,27$ reais. Evidencia-se que as internações por câncer de mama em mulheres na rede pública na Região Metropolitana de Porto Alegre têm repercutido no aumento de gastos e intensificação no uso de leitos hospitalares, tornando a assistência a esse tipo de agravo cada vez mais onerosa.

Pode-se inferir que as internações se caracterizaram por elevada incidência, curta duração e baixa letalidade na rede pública. Sugerem-se novas análises, principalmente acerca dos fluxos origem-destino das internações a fim de nortear o planejamento da assistência, visando melhoria da distribuição dos recursos.

\section{Referências}

1. INCA. Instituto Nacional de Câncer. A situação do câncer de mama no Brasil: síntese de dados dos sistemas de informação. Rio de Janeiro: INCA, 2019.

2. BRASIL. Ministério da Saúde. Estimativa 2020 - Incidência de câncer no Brasil. Rio de Janeiro: INCA, 2019.

3. IARC. International Agency for Research on Cancer. Latest global cancer data: Cancer burden rises to 18.1 million new cases and 9.6 million cancer deaths in 2018.2018.

4. BRASIL. Ministério da Saúde. Saúde Brasil 2014 - Uma análise da situação da situação de saúde e das causas extensas. Brasilia: Ministério da Saúde, 2015.

5. Barbosa IR, et al. Desigualdades regionais na mortalidade por câncer de colo de útero no brasil: Tendências e projeções até o ano 2030. Ciencia e Saude Coletiva, v. 21, n. 1, p. 253-262, 2016.

6. SIHSUS - Sistema de Informações Hospitalares do SUS. Datasus. Acessado em 02 de dezembro de 2020.

7. Ministério da Saúde. Departamento de Informática do SUS. Morbidade Hospitalar do SUS CID-10. Lista de Tabulação para Morbidade. Ministério da Saúde. Brasil. Acessado em 15 de dezembro de 2020. Disponível em: http://tabnet.datasus.gov.br/ cgi/sih/mxcid10lm.htm

8. Lessa, FJD, et al. Novas metodologias para vigilância epidemiológica: uso do Sistema de Informações Hospitalares - SIH/SUS. Informe Epidemiológico do Sus, v. 9, p. $3-27,2000$
9. Passos JG, Souza SRP. Análise da Mortalidade Hospitalar por Câncer de mama no Estado de São Paulo no período de 1999 a 2012. Science in Health. São Paulo: UNICID, 2010. v. 6, n. 2, p. 100-8,2015.

10. Oliveira $\mathrm{E}$, et al. Caderno Saúde Pública. Acesso à assistência oncológica: mapeamento dos fluxos origem-destino das internações e dos atendimentos ambulatoriais. 0 caso do câncer de mama, v.27, n.2, p.317-326, 2011.

11. Duarte DA P, Teixeira MTB. Social Iniquity and Mortality Related to Breastand Cervical Cancers: na Integrative Review / Iniquidade Social e Mortalidade por Câncer de Mama e Colo do Útero: Uma Revisão Integrativa. Revista de Pesquisa: Cuidado é Fundamental Online, v. 10, n. 3, p. 877, 2018.

12. Martins CA, et al. Evolução da Mortalidade por Câncer de Mama em Mulheres Jovens: Desafios para uma Política de Atenção Oncológica. Revista Brasileira de Cancerologia, v. 59, n. 3, p. 341-349, 2013.

13. Santos RS, Melo ECP. Internação por câncer de mama e colo de útero no Brasil. R. pesq.: cuid. fundam., v. 2, p. 217-219, 2010.

14. Almeida PF, et al. Desafios à coordenação dos cuidados em saúde: Estratégias de integração entre níveis assistenciais em grandes centros urbanos. Cadernos de Saude Publica, v. 26, n. 2, p. 286-298, 2010.

15. Lima AA. Densidade tecnológica e o cuidado humanizado em enfermagem: a realidade de dois serviços de saúde. 106 f. Dissertação (Mestrado em Saúde Coletiva). Instituto de Saúde Coletiva, Universidade Federal da bahia, Salvador, Bahia. 2013. 